\title{
Substituent effects on the in vitro and in vivo genotoxicity of 4-aminobiphenyl and 4-aminostilbene derivatives
}

\author{
Z. You, M.D. Brezzell, S.K. Das, B.H. Hooberman and J.E. Sinsheimer * \\ College of Pharmacy, University of Michigan, Ann Arbor, MI 48109-1065, USA \\ (Received 11 December 1992) \\ (Revision received 28 June 1993) \\ (Accepted 7 July 1993)
}

\begin{abstract}
Keywords: Aromatic amines; Salmonella mutagenicity; Chromosomal aberrations, bone-marrow, in vivo; Quantitative structureactivity relationships; Substituent electronic effects; Partition coefficients
\end{abstract}

\section{Summary}

4-Amino-4'-substituted biphenyls and 4-aminostilbenes substituted in the $3^{\prime}$ or $4^{\prime}$ position were studied for their in vitro and in vivo genotoxicity. The in vitro mutagenicity of the biphenyls with and without S9 activation was established with Salmonella strains TA98 and TA100 and that of the stilbenes with the same strains plus TA98/1,8-DNP ${ }_{6}$. The in vivo genotoxicity assay with both series of compounds was for chromosomal aberrations in the bone-marrow cells of mice following intraperitoneal administration of the chemicals. Hammett values of substituents, partition coefficients and frontier orbital energies ( $E_{\text {LUMO }}$ and $E_{\text {HOMO }}$ ) of the compounds were used for correlations with mutagenicity. The Salmonella mutagenicity in TA98 and TA98/1,8-DNP 6 with $\mathrm{S} 9$ was correlated to Hammett $\sigma^{+}$values for the 4-aminostilbene substituents, showing a strong trend of increasing mutagenicity with an increase in the electron-withdrawing capability of the substituent. Hydrophobicity of the stilbenes, however, had little effect on their relative mutagenicity. The 4-aminobiphenyls showed a correlation between their mutagenicity and Hammett $\sigma^{+}$values of their $4^{\prime}$-substituents in stain TA98 with S9, although the trend was not as strong as for the stilbenes. But unlike the stilbenes, TA98 mutagenicity of the biphenyls could also be correlated to hydrophobicity, and structure-activity correlations for the biphenyls was substantially improved when both $\sigma^{+}$and hydrophobicity data were included. For strain TA100 with S9, little correlation was found between mutagenicity of the stilbenes and any of the parameters. However, a limited correlation did exist between the mutagenicity of the biphenyls and their hydrophobicity. There was also limited correlations of the mutagenicity for the stilbenes in TA98 and TA98/1,8-DNP 6 with S9 to $E_{\text {LUMo }}$ or $E_{\text {HOMO. }}$. The in vivo genotoxicity results for the biphenyls and stilbenes could not be correlated to electronic effects as for the in vitro results, nor could they be explained by hydrophobicity. However, it is interesting to note that $3^{\prime}$-substituted 4-aminostilbenes were all substantially more genotoxic in vivo than their corresponding $4^{\prime}$-substituted counterparts. The most genotoxic compound in vivo in either series was 4-aminostilbene which would not have been predicted from the in vitro results.

\footnotetext{
* Corresponding author.
} 
As a continuation of our studies on structuregenotoxicity relationships, we have directed our attention to the electronic effects of substituents on the genotoxicity of benzidine and related aromatic amines. We previously described the substituent-electronic effects in a series of 3,3'-disubstituted benzidines (You et al., 1993). A prior study (Sinsheimer et al., 1992) indicated that the Salmonella strain TA98 mutagenicity of a limited number of 4-aminobiphenyl and 4-aminostilbene derivatives was dependent upon the electronwithdrawing ability of their $4^{\prime}$-substituents. The present study extends the number of amines under investigation in developing quantitative structure-activity relationships (QSAR) for these two series of compounds.

It is well established (Veschamber and Kergomard, 1966; Byron et al., 1966; Zheltov et al., 1970) that basicity for 4-aminobiphenyl and 4aminostilbene derivatives is highly correlated to the electronic effects of their substituents as measured by Hammett $\sigma$ values. Debnath et al. (1992), in their QSAR study for aromatic amines, have shown correlations of mutagenicity with frontier orbital energy and hydrophobicity. Our preceding paper (You et al., 1993) revealed a correlation between the Salmonella mutagenicity of 3,3'-disubstituted benzidines and their basicity, which we assigned to an increase in mutagenicity due to an increase in electron-withdrawing ability of the benzidine substituents. Thus, a unified rational could emerge to account for Salmonella mutagenicity in strain TA98 for the benzidines, the 4-aminobiphenyls and the 4-aminostilbenes. It is of special interest to conduct a QSAR study for these compounds undergoing complex processes of enzymatic activation, diffusion and DNA binding. It is also our continued objective to compare these in vitro relationships to an in vivo measure of genotoxicity, where it would be expected that the transport considerations and metabolic pathways would be more complex than in vitro. The in vivo test employed was an evaluation of chromosomal aberrations (CA) in bonemarrow cells of mice following intraperitoneal (i.p.) administration of the amines. This CA assay is a well-established short-term in vivo genotoxicity test on rapidly dividing cells (Preston et al., $1987)$ and its use is in agreement with the recom- mendations of Natarajan and Obe (1986) and Shelby (1988) that the detection of point mutations by the Ames Salmonella assay be supplemented by in vivo evaluations that respond to strand breaks.

\section{Materials and methods}

\section{Chemicals}

Test compounds in the 4-aminobiphenyl series included 4-amino-, 4-amino-4'-chloro-, 4-amino4'-nitro-, 4,4'-diamino-, 4-amino-4'-cyano-, 4amino-4'-iodo-, 4-amino-4'-hydroxy- and 4amino-4'-methylbiphenyl. Compounds in the 4aminostilbene series were 4-amino-, 4-amino-4'chloro-, 4-amino-4'-nitro-, 4,4'-diamino-, 4amino-3'-chloro-, 4-amino-3'-cyano-, 4-amino-4'cyano-, 4-amino-3'-methoxy-, 4-amino-4'methoxy-, 4-amino-4'-methyl- and 3,4'-diaminostilbene. The first four compounds in each of the two series were previously reported (Sinsheimer et al., 1992), while given below are the syntheses and characterizations of the remaining test compounds. Proton NMR spectra were recorded at $300 \mathrm{MHz}$ with $\mathrm{CDCl}_{3}$ as solvent except where indicated.

4-Amino-4'-cyanobiphenyl. 4-Cyano-4'-nitrobiphenyl was obtained by treatment of 4-amino4 '-nitrobiphenyl with nitrous acid followed by cuprous cyanide (Zheltov et al., 1970). The cyanobiphenyl was reduced through the Zinin procedure as previously described (Sinsheimer et al., 1992) to yield 4-amino-4'-cyanobiphenyl as a yellow powder (95\% for the reduction) after silica gel column separation (2:1 hexanes/ethyl acetate): $\mathrm{mp} 181-183.5^{\circ} \mathrm{C}$ (Zheltov et al., 1970: $\mathrm{mp}$ 182-184 $\left.{ }^{\circ} \mathrm{C}\right)$. NMR: $7.636\left(4 \mathrm{H}, \mathrm{AB}_{\mathrm{q}}, \mu_{\mathrm{AB}}=13 \mathrm{~Hz}\right.$, $\left.J_{\mathrm{AB}}=8.4 \mathrm{~Hz}\right), 7.425(2 \mathrm{H}, \mathrm{d}, J=8.4 \mathrm{~Hz}), 6.766$ $(2 \mathrm{H}, \mathrm{d}, J=8.4 \mathrm{~Hz}), 3.846(2 \mathrm{H}, \mathrm{br} \mathrm{s})$.

4-Amino-4'-hydroxybiphenyl. 4-Amino-4'nitrobiphenyl was treated with nitrous acid (1.1 molar equiv., $\mathrm{NaNO}_{2}+\mathrm{H}_{2} \mathrm{SO}_{4}$ ) in aqueous solution at $0^{\circ} \mathrm{C}$ for $40 \mathrm{~min}$. The resulting diazonium salt was then poured into boiling water and refluxed for $3 \mathrm{~h}$. The suspension after cooling was filtered. The solid obtained was treated with Zinin reagents and the resulting mixture poured into 
water. This aqueous system was extracted repeatedly with ether until no product was observed on TLC. The ether solution was then extracted with aqueous sodium hydroxide to remove the desired product from the organic phase. The basic solution was neutralized with aqueous $\mathrm{HCl}$ to a $\mathrm{pH}$ of 6.5 , and the precipitate filtered off. The resulting solid was crystallized from methanol to give a grey powder (48\% from 4-amino-4'-nitrobiphenyl): $\mathrm{mp}$ (dec.) $\sim 270^{\circ} \mathrm{C}$ (Czerwinska-Fejgin and Polaczkowa, 1967: mp 269-271 ${ }^{\circ} \mathrm{C}$ ). NMR (DMSO-d $\left.{ }_{6}\right): 9.266(1 \mathrm{H}, \mathrm{s}), 7.306(2 \mathrm{H}, \mathrm{d}, J=8.5$ $\mathrm{Hz}), 7.220(2 \mathrm{H}, \mathrm{d}, J=8.5 \mathrm{~Hz}), 6.745(2 \mathrm{H}, \mathrm{d}$, $J=8.5 \mathrm{~Hz}), 6.579(2 \mathrm{H}, \mathrm{d}, J=8.5 \mathrm{~Hz}), 5.054(2 \mathrm{H}$, br s).

4-Amino-4'-iodobiphenyl. 4-Iodo-4'-nitrobiphenyl was prepared by treating biphenyl (Aldrich, Milwaukee, WI) with iodine and nitric acid (Harley-Mason and Mann, 1940). Reduction of this nitrobiphenyl with moist iron powder (Zheltov et al., 1970) gave 4-amino-4'-iodobiphenyl: $\mathrm{mp}$ $160-161^{\circ} \mathrm{C}$ (Zheltov et al., 1970: $\mathrm{mp} 161-161.5^{\circ} \mathrm{C}$ ). NMR: $7.699(2 \mathrm{H}, \mathrm{d}, J=8.5 \mathrm{~Hz}), 7.368(2 \mathrm{H}, \mathrm{d}$, $J=8.7 \mathrm{~Hz}), 7.269(2 \mathrm{H}, \mathrm{d}, J=8.5 \mathrm{~Hz}), 6.745(2 \mathrm{H}$, $\mathrm{d}, J=8.7 \mathrm{~Hz}), 3.761(2 \mathrm{H}$, br s).

4-Amino-4'-methylbiphenyl. 4-Methylbiphenyl (Aldrich) was treated with nitric acid/acetic acid to yield 4-methyl-4'-nitrobiphenyl (Grieve and Hey, 1932). Reduction of this product through the Zinin procedure gave 4-amino-4'-methylbiphenyl after silica gel column separation (2:1 hexanes/ethyl acetate) and crystallization from methanol/water $(77 \%$ for the reduction): $\mathrm{mp}$ 97-98 ${ }^{\circ} \mathrm{C}$ (Grieve and Hey, 1932: $\mathrm{mp} 97^{\circ} \mathrm{C}$ ). NMR: $7.426(2 \mathrm{H}, \mathrm{d}, J=8.0 \mathrm{~Hz}), 7.391(2 \mathrm{H}, \mathrm{d}, J=8.5$ $\mathrm{Hz}), 7.199(2 \mathrm{H}, \mathrm{d}, J=8.0 \mathrm{~Hz}), 6.742(2 \mathrm{H}, \mathrm{d}$, $J=8.5 \mathrm{~Hz}), 3.695(2 \mathrm{H}$, br s), $2.368(3 \mathrm{H}, \mathrm{s})$.

The 4-aminostilbenes were synthesized through an established procedure (Sinsheimer et al., 1992) with the modification that Wittig reaction products were first filtered through silica gel to remove polar impurities before the $\mathrm{Z}-\mathrm{E}$ double bond isomerization. 4-Amino-3'-methoxy- and 4amino-4'-methyl-stilbene were purified by silica gel column chromatography with hexanes/ethyl acetate solvent systems, while 4-amino-3'-chloro-, 4-amino-3'-cyano-, 4-amino-4'-cyano-, 4-amino- 4'-methoxy- and 3,4'-diamino-stilbene by crystallization from alcoholic solvent systems. Yields given below are for the Zinin reduction products.

3,4'-Diaminostilbene. 95\%; $\mathrm{mp}(\mathrm{MeOH} /$ Water) $155-156^{\circ} \mathrm{C}$ (Ashley et al., 1942: $153^{\circ} \mathrm{C}$ ); NMR $7.319(2 \mathrm{H}, \mathrm{d}, J=8.5 \mathrm{~Hz}), 7.125(1 \mathrm{H}$, dd, $J=7.8,7.7 \mathrm{~Hz}), 6.978(1 \mathrm{H}, \mathrm{d}, J=16.3 \mathrm{~Hz}), 6.890$ $(1 \mathrm{H}$, br d, $J=7.7 \mathrm{~Hz}), 6.830(1 \mathrm{H}, \mathrm{d}, J=16.3 \mathrm{~Hz})$, $6.807(1 \mathrm{H}, \mathrm{dd}, J=2.2,1.6 \mathrm{~Hz}), 6.669(2 \mathrm{H}, \mathrm{d}$, $J=8.5 \mathrm{~Hz}), 6.562(1 \mathrm{H}$, ddd, $J=7.8,2.2,1.6 \mathrm{~Hz})$, $3.699(4 \mathrm{H}$, br s).

4-Amino-3'-cyanostilbene. $80 \%$; $\mathrm{mp}(\mathrm{EtOH})$ $177-178^{\circ} \mathrm{C}$; NMR $7.722(1 \mathrm{H}$, br s), $7.660(1 \mathrm{H}, \mathrm{dt}$, $J=7.6,1.6 \mathrm{~Hz}), 7.467(1 \mathrm{H}, \mathrm{dt}, J=7.6,1.5 \mathrm{~Hz})$, $7.411(1 \mathrm{H}, \mathrm{t}, J=7.6 \mathrm{~Hz}), 7.341(2 \mathrm{H}, \mathrm{d}, J=8.5$ $\mathrm{Hz}), 7.060(1 \mathrm{H}, \mathrm{d}, J=16.4 \mathrm{~Hz}), 6.856(1 \mathrm{H}, \mathrm{d}$, $J=16.4 \mathrm{~Hz}), 6.686(2 \mathrm{H}, \mathrm{d}, J=8.5 \mathrm{~Hz}), 3.809(2 \mathrm{H}$, br s); $\mathrm{C}_{15} \mathrm{H}_{12} \mathrm{~N}_{2}$ calculated C $81.79 \%$, H 5.49\%, $\mathrm{N} 12.72 \%$; found C $81.53 \%$, H 5.28\%, N $12.32 \%$.

4-Amino-4'-cyanostilbene. $84 \% ; \mathrm{mp}(\mathrm{EtOH})$ 225-227 $7^{\circ} \mathrm{C}$ (Hanna et al., 1980: $226-228^{\circ} \mathrm{C}$ ); NMR $7.592(2 \mathrm{H}, \mathrm{d}, J=8.4 \mathrm{~Hz}), 7.516(2 \mathrm{H}, \mathrm{d}, J=8.4$ $\mathrm{Hz}), 7.348(2 \mathrm{H}, \mathrm{d}, J=8.4 \mathrm{~Hz}), 7.120(1 \mathrm{H}, \mathrm{d}$, $J=16.3 \mathrm{~Hz}), 6.883(1 \mathrm{H}, \mathrm{d}, J=16.3 \mathrm{~Hz}), 6.680$ $(1 \mathrm{H}, \mathrm{d}, J=8.4 \mathrm{~Hz}), 3.835(2 \mathrm{H}, \mathrm{br} \mathrm{s})$.

4-Amino-3'-chlorostilbene. $87 \% ; \mathrm{mp}(\mathrm{EtOH})$ $144-145^{\circ} \mathrm{C}$ (Veschamber and Kergomard, 1966: 143.5-144.5 $\left.5^{\circ} \mathrm{C}\right)$; NMR $7.451(1 \mathrm{H}$, br s), $7.327(3 \mathrm{H}$, $\mathrm{d}, J=8.5 \mathrm{~Hz}), 7.248(1 \mathrm{H}, \mathrm{t}, J=7.7 \mathrm{~Hz}), 7.168$ $(1 \mathrm{H}$, br d, $J=7.6 \mathrm{~Hz}), 7.018(1 \mathrm{H}, \mathrm{d}, J=16.3 \mathrm{~Hz})$, $6.836(1 \mathrm{H}, \mathrm{d}, J=16.3 \mathrm{~Hz}), 6.674(2 \mathrm{H}, \mathrm{d}, J=8.5$ $\mathrm{Hz}), 3.775$ (2H, br s).

4-Amino-3'-methoxystilbene. 98\%; mp 61.5$62.5^{\circ} \mathrm{C}$; NMR $7.339(2 \mathrm{H}, \mathrm{d}, J=8.5 \mathrm{~Hz}), 7.252$ $(1 \mathrm{H}, \mathrm{t}, J=8.0 \mathrm{~Hz}), 7.071(1 \mathrm{H}, \mathrm{d}, J=8.0 \mathrm{~Hz})$, $7.024(1 \mathrm{H}, \mathrm{d}, J=16.3 \mathrm{~Hz}), 7.010(1 \mathrm{H}$, br s), 6.890 $(1 \mathrm{H}, \mathrm{d}, J=16.3 \mathrm{~Hz}), 6.776(1 \mathrm{H}, \mathrm{dd}, J=8.0,1.8$ $\mathrm{Hz}), 6.680(2 \mathrm{H}, \mathrm{d}, J=8.5 \mathrm{~Hz}), 3.844(3 \mathrm{H}, \mathrm{s}), 3.76$ $\left(2 \mathrm{H}\right.$, br s); $\mathrm{C}_{15} \mathrm{H}_{15} \mathrm{NO}$ calculated $\mathrm{C} 79.97 \%, \mathrm{H}$ $6.71 \%, \mathrm{~N} 6.22 \%$; found $\mathrm{C} 79.70 \%, \mathrm{H} 6.67 \%, \mathrm{~N}$ $6.00 \%$.

4-Amino-4'-methoxystilbene. $80 \%$; $\mathrm{mp}(\mathrm{EtOH})$ $174-175^{\circ} \mathrm{C}$ (Veschamber and Kergomard, 1966: 
TABLE 1

MUTAGENICITY OF 4-AMINOSTILBENES IN Salmonella typhimurium a

\begin{tabular}{|c|c|c|c|c|c|c|}
\hline \multirow{2}{*}{$\begin{array}{l}\text { Compounds } \\
\text { ( } \mu \text { moles) }\end{array}$} & \multicolumn{2}{|l|}{ TA98 } & \multicolumn{2}{|c|}{ TA98/1,8-DNP 6} & \multicolumn{2}{|l|}{ TA100 } \\
\hline & $-\mathrm{S} 9$ & $+S 9$ & $-\$ 9$ & $+\mathrm{S} 9$ & $-\mathrm{S} 9$ & $+\mathrm{S} 9$ \\
\hline \multicolumn{7}{|c|}{$4,4^{\prime}$-Diaminostilbene } \\
\hline 0 & $29 \pm 6$ & $45 \pm 7$ & $38 \pm 10$ & $47 \pm 11$ & $121 \pm 14$ & $128 \pm 17$ \\
\hline 0.01 & & & $39 \pm 5$ & $51 \pm 4$ & & \\
\hline 0.05 & $25 \pm 4$ & $64 \pm 3$ & & & $121 \pm 6$ & $152 \pm 15$ \\
\hline 0.1 & $34 \pm 7$ & $96 \pm 15$ & $27 \pm 7$ & $44 \pm 14$ & $121 \pm 14$ & $144 \pm 24$ \\
\hline 0.3 & & & $24 \pm 7$ & $44 \pm 6$ & & \\
\hline 0.33 & $27 \pm 11$ & $146 \pm 19^{b}$ & & & $117 \pm 10$ & $173 \pm 18$ \\
\hline 0.5 & & & $39 \pm 6$ & $55 \pm 1$ & & \\
\hline 0.6 & & & $25 \pm 2$ & $40 \pm 5$ & & \\
\hline 0.66 & $35 \pm 8$ & $159 \pm 35$ & & & $131 \pm 14$ & $150 \pm 26$ \\
\hline 1 & $34 \pm 7$ & $142 \pm 16$ & $34 \pm 5$ & $52 \pm 9$ & $109 \pm 20$ & $167 \pm 18$ \\
\hline 3 & $28 \pm 9$ & $118 \pm 10$ & $27 \pm 10$ & $41 \pm 8^{b}$ & & $139 \pm 18^{b}$ \\
\hline \multicolumn{7}{|c|}{ 4-Amino-4'-methoxystilbene } \\
\hline 0 & $30 \pm 7$ & $53 \pm 12$ & $25 \pm 12$ & $35 \pm 8$ & $99 \pm 13$ & $118 \pm 14$ \\
\hline 0.01 & $34 \pm 2$ & $55 \pm 9$ & & & $108 \pm 23$ & $130 \pm 12$ \\
\hline 0.033 & $48 \pm 7$ & $56 \pm 12$ & & & $108 \pm 14$ & $149 \pm 6$ \\
\hline 0.05 & $44 \pm 8$ & $66 \pm 5$ & & & $124 \pm 20$ & $154 \pm 21$ \\
\hline 0.066 & $38 \pm 7$ & $87 \pm 9$ & & & $96 \pm 8$ & $167 \pm 10$ \\
\hline 0.1 & $51 \pm 10$ & $84 \pm 7$ & $24 \pm 5$ & $42 \pm 6$ & $117 \pm 17$ & $193 \pm 29$ \\
\hline 0.3 & $51 \pm 14$ & $163 \pm 30$ & $24 \pm 7$ & $46 \pm 9$ & $128 \pm 7$ & $529 \pm 17$ \\
\hline 0.33 & $49 \pm 5$ & $158 \pm 21^{b}$ & & & $142 \pm 16$ & $546 \pm 84$ \\
\hline 0.6 & & & $25 \pm 8$ & $50 \pm 6$ & & \\
\hline 0.66 & $60 \pm 22$ & $218 \pm 22$ & & & $141 \pm 20$ & $879 \pm 109^{b}$ \\
\hline 1 & $56 \pm 5$ & $237 \pm 25$ & $24 \pm 4$ & $58 \pm 9^{b}$ & $143 \pm 12$ & $815 \pm 56$ \\
\hline 3 & & & $27 \pm 10$ & $66 \pm 16$ & & \\
\hline \multicolumn{7}{|c|}{ 4-Amino-4'-methylstilbene } \\
\hline 0 & $27 \pm 5$ & $40 \pm 10$ & $26 \pm 9$ & $25 \pm 7$ & $107 \pm 27$ & $110 \pm 17$ \\
\hline 0.01 & $37 \pm 7$ & $141 \pm 27$ & $31 \pm 6$ & $83 \pm 10$ & $100 \pm 13$ & $218 \pm 49$ \\
\hline 0.033 & $38 \pm 4$ & $301 \pm 39$ & $41 \pm 8$ & $122 \pm 45$ & $125 \pm 17$ & $436 \pm 58$ \\
\hline 0.066 & $44 \pm 6$ & $507 \pm 44^{b}$ & $46 \pm 20$ & $200 \pm 27$ & $116 \pm 26$ & $879 \pm 78$ \\
\hline 0.1 & $34 \pm 12$ & $654 \pm 78$ & $41 \pm 13$ & $284 \pm 39^{b}$ & $101 \pm 8$ & $1165 \pm 141^{b}$ \\
\hline 0.2 & & & $27 \pm 7$ & $405 \pm 47$ & & \\
\hline 0.3 & $42 \pm 5$ & $924 \pm 194$ & $52 \pm 2$ & $602 \pm 38$ & $126 \pm 12$ & $1683 \pm 150$ \\
\hline \multicolumn{7}{|c|}{ 3,4'-Diaminostilbene } \\
\hline 0 & $28 \pm 5$ & $45 \pm 17$ & $38 \pm 10$ & $47 \pm 10$ & $130 \pm 18$ & $128 \pm 17$ \\
\hline 0.005 & $31 \pm 10$ & $71 \pm 13$ & & & $128 \pm 15$ & $235 \pm 17$ \\
\hline 0.01 & $27 \pm 9$ & $83 \pm 12$ & $28 \pm 11$ & $64 \pm 8$ & $127 \pm 10$ & $318 \pm 25$ \\
\hline 0.02 & $26 \pm 10$ & $126 \pm 8$ & & & $134 \pm 23$ & $574 \pm 25$ \\
\hline 0.03 & & & $27 \pm 8$ & $92 \pm 11$ & & \\
\hline 0.033 & $36 \pm 12$ & $215 \pm 22$ & & & $135 \pm 8$ & $1105 \pm 64$ \\
\hline 0.066 & $38 \pm 16$ & $363 \pm 34^{b}$ & & & $120 \pm 12$ & $2063 \pm 132^{b}$ \\
\hline 0.1 & $29 \pm 11$ & $325 \pm 51$ & $39 \pm 8$ & $221 \pm 71^{b}$ & $145 \pm 18$ & $2425 \pm 221$ \\
\hline 0.3 & & & $28 \pm 10$ & $332 \pm 37$ & & \\
\hline 0.5 & $47 \pm 13$ & & $51 \pm 6$ & $445 \pm 30$ & $123 \pm 11$ & \\
\hline 1 & $70 \pm 13$ & & $51 \pm 10$ & $176 \pm 93$ & $176 \pm 13$ & \\
\hline \multicolumn{7}{|c|}{ 4-Aminostilbene } \\
\hline 0 & $30 \pm 5$ & $43 \pm 7$ & $33 \pm 12$ & $43 \pm 13$ & $130 \pm 18$ & $128 \pm 17$ \\
\hline 0.01 & $30 \pm 8$ & $58 \pm 10$ & $30 \pm 10$ & $51 \pm 9$ & $124 \pm 10$ & $156 \pm 12$ \\
\hline 0.03 & & & $18 \pm 4$ & $65 \pm 10$ & & \\
\hline 0.033 & $30 \pm 9$ & $131 \pm 30$ & & & $115 \pm 14$ & $318 \pm 32$ \\
\hline
\end{tabular}


TABLE 1 (continued)

\begin{tabular}{|c|c|c|c|c|c|c|}
\hline \multirow{2}{*}{$\begin{array}{l}\text { Compounds } \\
\text { ( } \mu \text { moles) }\end{array}$} & \multicolumn{2}{|l|}{ TA98 } & \multicolumn{2}{|c|}{ TA98/1,8-DNP 6} & \multicolumn{2}{|l|}{ TA100 } \\
\hline & $-\mathrm{S} 9$ & $+\mathrm{S} 9$ & -S9 & $+\mathrm{S} 9$ & $-\mathrm{S9}$ & $+S 9$ \\
\hline \multicolumn{7}{|c|}{ 4-Aminostilbene } \\
\hline 0.066 & $32 \pm 8$ & $298 \pm 46$ & & & $112 \pm 17$ & $741 \pm 65$ \\
\hline 0.1 & $41 \pm 11$ & $391 \pm 77^{b}$ & $31 \pm 14$ & $133 \pm 7$ & $135 \pm 7$ & $1094 \pm 85^{b}$ \\
\hline 0.3 & $42 \pm 5$ & $500 \pm 118$ & $27 \pm 4$ & $438 \pm 12$ & $155 \pm 21$ & $2277 \pm 153$ \\
\hline 0.33 & $51 \pm 6$ & & & & & \\
\hline 0.5 & $41 \pm 2$ & & $50 \pm 4$ & $501 \pm 3^{b}$ & $112 \pm 17$ & \\
\hline 0.66 & $49 \pm 5$ & & & & & \\
\hline 1 & $70 \pm 5$ & & $42 \pm 16$ & $796 \pm 116$ & $178 \pm 17$ & \\
\hline 3 & $64 \pm 8$ & & & & & \\
\hline \multicolumn{7}{|c|}{ 4-Amino-3'-methoxystilbene } \\
\hline 0 & $21 \pm 6$ & $37 \pm$ & $40 \pm 27$ & $50 \pm 23$ & $119 \pm 21$ & $148 \pm 20$ \\
\hline 0.003 & & & $12 \pm 4$ & $32 \pm 8$ & & \\
\hline 0.01 & $25 \pm 7$ & $56 \pm 19$ & $39 \pm 21$ & $56 \pm 21$ & $123 \pm 26$ & $150 \pm 21$ \\
\hline 0.03 & & & $37 \pm 22$ & $77 \pm 26$ & & \\
\hline 0.033 & $41 \pm 26$ & $156 \pm 59$ & & & $125 \pm 16$ & $304 \pm 51$ \\
\hline 0.066 & $39 \pm 29$ & $394 \pm 70$ & & & $129 \pm 21$ & $805 \pm 144$ \\
\hline 0.1 & $43 \pm 31$ & $526 \pm 51^{b}$ & $40 \pm 24$ & $185 \pm 28$ & $142 \pm 24$ & $1459 \pm 128$ \\
\hline 0.2 & $60 \pm 16$ & $341 \pm 17$ & & & $154 \pm 7$ & $2183 \pm 103^{b}$ \\
\hline 0.3 & & & $40 \pm 26$ & $479 \pm 68^{b}$ & & \\
\hline 0.33 & $18 \pm 5$ & $103 \pm 32$ & & & $123 \pm 25$ & $1271 \pm 155$ \\
\hline 1 & & & $27 \pm 5$ & $359 \pm 47$ & & \\
\hline \multicolumn{7}{|c|}{ 4-Amino-4'-chlorostilbene } \\
\hline 0 & $31 \pm 5$ & $50 \pm 10$ & $23 \pm 6$ & $30 \pm 11$ & $115 \pm 20$ & $123 \pm 17$ \\
\hline 0.005 & $30 \pm 6$ & $56 \pm 19$ & & & $136 \pm 13$ & $113 \pm 11$ \\
\hline 0.01 & $38 \pm 6$ & $73 \pm 14$ & $26 \pm 8$ & $57 \pm 9$ & $123 \pm 24$ & $162 \pm 32$ \\
\hline 0.03 & & & $36 \pm 10$ & $336 \pm 43$ & & \\
\hline 0.033 & $41 \pm 3$ & $297 \pm 24$ & & & $132 \pm 12$ & $285 \pm 31$ \\
\hline 0.05 & $44 \pm 10$ & $628 \pm 132$ & $29 \pm 8$ & $281 \pm 41$ & $112 \pm 15$ & $396 \pm 33$ \\
\hline 0.066 & $59 \pm 7$ & $932 \pm 98$ & & & $133 \pm 9$ & $611 \pm 71$ \\
\hline 0.1 & $46 \pm 11$ & $1496 \pm 117^{b}$ & $34 \pm 9$ & $742 \pm 97^{b}$ & $125 \pm 15$ & $792 \pm 70^{b}$ \\
\hline 0.3 & $57 \pm 11$ & $2152 \pm 68$ & $32 \pm 9$ & $972 \pm 89$ & $107 \pm 5$ & $900 \pm 25$ \\
\hline 0.33 & $34 \pm 2$ & & & & & \\
\hline 0.5 & $58 \pm 13$ & $2162 \pm 183$ & $35 \pm 7$ & $1023 \pm 69$ & $77 \pm 2$ & $895 \pm 14$ \\
\hline 0.66 & $38 \pm 3$ & & & & & \\
\hline 1 & $36 \pm 5$ & & $30 \pm 1$ & $977 \pm 93$ & & \\
\hline 3 & $40 \pm 5$ & & & & & \\
\hline \multicolumn{7}{|c|}{ 4-Amino-3'-chlorostilbene } \\
\hline 0 & $18 \pm 3$ & $28 \pm$ & $27 \pm 7$ & $37 \pm 3$ & $110 \pm 15$ & $115 \pm$ \\
\hline 0.003 & & & $30 \pm 2$ & $46 \pm 8$ & & \\
\hline 0.01 & $18 \pm 7$ & $37 \pm$ & $27 \pm 8$ & $57 \pm 7$ & $108 \pm 15$ & $131 \pm 15$ \\
\hline 0.03 & & & $32 \pm 6$ & $193 \pm 128$ & & \\
\hline 0.033 & $26 \pm 4$ & $162 \pm 25$ & & & $117 \pm 18$ & $209 \pm 18$ \\
\hline 0.06 & & & $32 \pm 4$ & $347 \pm 30$ & & \\
\hline 0.066 & $28 \pm 8$ & $621 \pm 41$ & $41 \pm 7$ & $570 \pm 58$ & $112 \pm 23$ & $371 \pm 34$ \\
\hline 0.1 & $32 \pm 10$ & $1043 \pm 38^{b}$ & $40 \pm 5$ & $783 \pm 244^{b}$ & $116 \pm 10$ & $627 \pm 42$ \\
\hline 0.3 & $43 \pm 9$ & $1393 \pm 92$ & $43 \pm 11$ & $1071 \pm 161$ & $125 \pm 12$ & $1085 \pm 32$ \\
\hline \multicolumn{7}{|c|}{ 4-Amino-3'-cyanostilbene } \\
\hline 0 & $28 \pm 6$ & $41 \pm \quad 9$ & $48 \pm 20$ & $53 \pm 20$ & $110 \pm 23$ & $130 \pm 15$ \\
\hline 0.003 & & & $29 \pm 4$ & $55 \pm 11$ & & \\
\hline 0.01 & & $90 \pm 14$ & $46 \pm 19$ & $95 \pm 26$ & $108 \pm 4$ & $151 \pm 15$ \\
\hline 0.02 & & $152 \pm 43$ & & & & $184 \pm 12$ \\
\hline 0.03 & & & $47 \pm 20$ & $282 \pm 156$ & & \\
\hline
\end{tabular}


TABLE 1 (continued)

\begin{tabular}{|c|c|c|c|c|c|c|}
\hline \multirow{2}{*}{$\begin{array}{l}\text { Compounds } \\
\text { ( } \mu \text { moles })\end{array}$} & \multicolumn{2}{|l|}{ TA98 } & \multicolumn{2}{|c|}{ TA98/1,8-DNP 6} & \multicolumn{2}{|l|}{ TA100 } \\
\hline & $-\mathrm{S9}$ & $+\mathbf{S} 9$ & $-S 9$ & $+\$ 9$ & $-\mathrm{S} 9$ & $+\mathrm{S} 9$ \\
\hline \multicolumn{7}{|c|}{ 4-Amino-3'-cyanostilbene } \\
\hline 0.033 & $34 \pm 6$ & $614 \pm 17$ & & & $114 \pm 14$ & $328 \pm 20$ \\
\hline 0.04 & & $718 \pm 193$ & & & & $314 \pm 31$ \\
\hline 0.06 & & $1507 \pm 337$ & $20 \pm 5$ & $732 \pm 75$ & & $663 \pm 128$ \\
\hline 0.066 & $36 \pm 5$ & $1869 \pm 117$ & & & $115 \pm 21$ & $1143 \pm 60$ \\
\hline 0.08 & & $2341 \pm 595$ & & & & $1151 \pm 224$ \\
\hline 0.1 & $39 \pm 9$ & $2743 \pm 19^{b}$ & $48 \pm 25$ & $1590 \pm 172$ & $114 \pm 30$ & $2412 \pm 123^{b}$ \\
\hline 0.3 & $107 \pm 12$ & $1478 \pm 372$ & $88 \pm 18$ & $3007 \pm 413^{b}$ & $140 \pm 20$ & $1239 \pm 108$ \\
\hline 0.33 & $83 \pm 12$ & & & & $126 \pm 28$ & \\
\hline 0.66 & $91 \pm 32$ & & & & $133 \pm 23$ & \\
\hline 1 & $100 \pm 14$ & & $69 \pm 7$ & $3670 \pm 318$ & $146 \pm 39$ & \\
\hline \multicolumn{7}{|c|}{ 4-Amino-4'-cyanostilbene } \\
\hline 0 & $22 \pm 4$ & $35 \pm 6$ & $27 \pm 7$ & $37 \pm 3$ & $97 \pm 16$ & $121 \pm 17$ \\
\hline 0.003 & & & $23 \pm 8$ & $52 \pm 7$ & & \\
\hline 0.005 & $17 \pm 3$ & $51 \pm 12$ & & & $107 \pm 4$ & $114 \pm 10$ \\
\hline 0.01 & $22 \pm 7$ & $56 \pm 13$ & $35 \pm 14$ & $57 \pm 12$ & $106 \pm 21$ & $132 \pm 20$ \\
\hline 0.02 & $24 \pm 6$ & $91 \pm 2$ & & & $125 \pm 6$ & $163 \pm 16$ \\
\hline 0.03 & $26 \pm 10$ & $127 \pm 28$ & $24 \pm 8$ & $159 \pm 76$ & $119 \pm 13$ & $175 \pm 11$ \\
\hline 0.033 & $27 \pm 5$ & $197 \pm 24$ & & & $114 \pm 18$ & $178 \pm 27$ \\
\hline 0.05 & $26 \pm 8$ & $242 \pm 26$ & & & $105 \pm 3$ & $181 \pm 4$ \\
\hline 0.06 & & & $22 \pm 5$ & $592 \pm 35$ & & \\
\hline 0.066 & $29 \pm 2$ & $699 \pm 79$ & $26 \pm 3$ & $676 \pm 162$ & $113 \pm 12$ & $274 \pm 27$ \\
\hline 0.1 & $25 \pm 12$ & $1529 \pm 120$ & $25 \pm 9$ & $1042 \pm 100^{b}$ & $109 \pm 14$ & $436 \pm 52$ \\
\hline 0.3 & $24 \pm 7$ & $2322 \pm 121^{b}$ & $26 \pm 3$ & $1098 \pm 100$ & $118 \pm 13$ & $788 \pm 54^{b}$ \\
\hline 0.5 & $34 \pm 11$ & & & & & \\
\hline 1 & $25 \pm 5$ & & & & & \\
\hline \multicolumn{7}{|c|}{ 4-Amino-4'-nitrostilbene } \\
\hline 0 & $33 \pm 6$ & $53 \pm 13$ & $62 \pm 12$ & $69 \pm 11$ & $107 \pm 20$ & $123 \pm 24$ \\
\hline 0.001 & $48 \pm 4$ & $68 \pm \quad 8$ & $63 \pm 6$ & $85 \pm 10$ & $88 \pm 14$ & $122 \pm 21$ \\
\hline 0.003 & & & $72 \pm 17$ & $76 \pm 13$ & & \\
\hline 0.005 & $46 \pm 12$ & $160 \pm 25$ & $74 \pm 1$ & $107 \pm 12$ & $120 \pm 9$ & $158 \pm 27$ \\
\hline 0.01 & $68 \pm 14$ & $304 \pm 23$ & $72 \pm 7$ & $155 \pm 49$ & $119 \pm 17$ & $183 \pm 23$ \\
\hline 0.02 & $78 \pm 13$ & $748 \pm 61$ & & & $139 \pm 17$ & $257 \pm 29$ \\
\hline 0.03 & $75 \pm 15$ & $1320 \pm 178$ & $77 \pm 8$ & $394 \pm 11$ & $130 \pm 38$ & $334 \pm 42$ \\
\hline 0.033 & $114 \pm 11$ & $1685 \pm 202$ & & & $111 \pm 9$ & $326 \pm 23$ \\
\hline 0.05 & $90 \pm 15$ & $2042 \pm 264$ & $86 \pm 6$ & $916 \pm 26^{b}$ & $121 \pm 14$ & $432 \pm 62^{b}$ \\
\hline 0.066 & $106 \pm 5$ & $2637 \pm 87^{b}$ & & & $98 \pm 14$ & $363 \pm 18$ \\
\hline 0.1 & $113 \pm 16$ & $2105 \pm 259$ & $92 \pm 6$ & $839 \pm 46$ & $121 \pm 13$ & $477 \pm 70$ \\
\hline 0.3 & & & $94 \pm 8$ & $1021 \pm 187$ & & \\
\hline 0.33 & $155 \pm 10$ & & & & & \\
\hline 0.66 & $166 \pm 16$ & & & & & \\
\hline 1 & $199 \pm 24$ & & & & & \\
\hline
\end{tabular}

a Combined data from at least two independent experiments, with triplicate plating per dose and at least 5 doses per experiment.

${ }^{b}$ Indicates highest dose included in linear regression analysis.

$\left.172-173^{\circ} \mathrm{C}\right)$; NMR $7.403(2 \mathrm{H}, \mathrm{d}, J=8.4 \mathrm{~Hz}), 7.307$ $(2 \mathrm{H}, \mathrm{d}, J=8.0 \mathrm{~Hz}), 6.880(2 \mathrm{H}, \mathrm{s}), 6.871(2 \mathrm{H}, \mathrm{d}$, $J=8.0 \mathrm{~Hz}), 6.668(2 \mathrm{H}, \mathrm{d}, J=8.4 \mathrm{~Hz}), 3.820(3 \mathrm{H}$, s), $3.72(2 \mathrm{H}$, br s).
4-Amino-4'-methylstilbene. 92\%; mp 158$159^{\circ} \mathrm{C}$ (Veschamber and Kergomard, 1966: $\left.150^{\circ} \mathrm{C}\right)$; NMR $7.365(2 \mathrm{H}, \mathrm{d}, J=8.0 \mathrm{~Hz}), 7.321$ $(2 \mathrm{H}, \mathrm{d}, J=8.4 \mathrm{~Hz}), 7.136(2 \mathrm{H}, \mathrm{d}, J=8.0 \mathrm{~Hz})$, 
$6.970(1 \mathrm{H}, \mathrm{d}, J=16.4 \mathrm{~Hz}), 6.890(1 \mathrm{H}, \mathrm{d}, J=16.4$ $\mathrm{Hz}), 6.670(2 \mathrm{H}, \mathrm{d}, J=8.4 \mathrm{~Hz}), 3.72(2 \mathrm{H}$, br s), $2.342(3 \mathrm{H}, \mathrm{s})$.

\section{Mutagenicity assays}

Compounds dissolved in DMSO were tested for concentration-mutagenicity relationships using Salmonella typhimurium strains TA100 and TA98 kindly provided by Dr. B.N. Ames, University of California, Berkeley. In addition, the 1,8dinitropyrene resistant strain TA98/1,8-DNP 6 (McCoy et al., 1981) kindly provided by Dr. H.S. Rosenkranz, University of Pittsburgh was used. The standard plate incorporation assay with and without metabolic activation as described by Maron and Ames (1983) was used. Plates were scored with an automatic counter (Autocount, Dynatech Labs, Chantilly, VA) which had been calibrated against manually scored plates. Each dose was run in triplicate and the dose-response relationship confirmed in a second set of tests. Linear regression analysis was applied to the initial portion of the dose-response relationships to obtain revertants/ $\mu$ mole for comparing mutagenic potencies.

\section{Chromosomal aberrations}

Test chemicals were administered i.p. in DMSO $(2 \mathrm{ml} / \mathrm{kg})$ as single $100 \mathrm{mg} / \mathrm{kg}$ doses to each of 4 mice. Negative control mice were injected with $2 \mathrm{ml} / \mathrm{kg}$ DMSO while DMBA was used as a positive control at a dose of $100 \mathrm{mg} / \mathrm{kg}$ in DMSO. The dose for the test compounds is based upon our concentration-response data reported for benzidine (Sinsheimer et al., 1992). CA assays were conducted as previously described (Giri et al.,1989) with a scoring time of 24 $h$ after injection which is consistent with the protocol of Preston et al. (1987). This scoring time was found to be an optimal time for benzidine after testing at 6,12 and $24 \mathrm{~h}$. All the slides were coded and observed by a single observer where 100 well-spread metaphase cells were scored per animal from each of 4 animals at each concentration tested. Mitotic indices were calculated from 1000 cells/animal and expressed as percentages. The aberration frequencies per cell for chromatid and chromosome types were calcu- lated. Gaps were recorded but not included in the frequency of aberrations per cell.

\section{Results and discussions}

\section{In vitro results}

The 4-aminostilbenes (Table 1) showed mutagenic activity in TA98 and TA100 with S9 activation and no or minor activity without such activation. This pattern of mutagenic activity is consistent with the well established metabolic transformation of aromatic amines by the liver to produce DNA alkylating agents (Beland and Kadlubar, 1985, 1990). In addition, all the 4-aminostilbenes with the exception of the 4 '-cyano derivative had reduced potency in TA98/1,8$\mathrm{DNP}_{6}$ vs. TA98 with $\mathrm{S} 9$ activation, indicating a positive role for the bacterial transacetylase enzyme (Saito et al., 1985) in the activation process. There is a high correlation $\left(r^{2}=0.85\right)$ of the mutagenic responses between the two TA98 strains. In the case of $3,4^{\prime}$-diaminostilbene, the regioselectivity of amino group activation is unknown, but for the purpose of the correlation the 3-amino is assumed to be the substituent and the 4 '-amino the activation center.

The 4-aminobiphenyls (Table 2) showed similar results in TA98 and TA100 in that S9 activation was required for mutagenicity, with the exception of 4-amino-4'-nitrobiphenyl. The nitro derivative exhibited considerable potency in TA98 without S9, which is in agreement with previous reports for that compound (Nohara et al., 1985) and for nitroaromatics in general (Rosenkranz and Mermelstein, 1985).

Table 3 lists the physicochemical parameters for both series of amines, including Hammett $\sigma$ and $\sigma^{+}$values for the meta' and para' substituents of the test compounds, frontier orbital energies (lowest unoccupied and highest occupied molecular orbitals; $E_{\mathrm{LUMO}}$ and $\left.E_{\mathrm{HOMO}}\right)$ and partition coefficients $\left(\pi_{\text {HPLC }}\right)$. Also given in this table are the slopes (revertants $/ \mu$ mole) for the mutagenicity testing in the presence of $S 9$ from Tables 1 and 2 . There was little correlation of the mutagenicity in TA100 with either electron-donating/ withdrawing ability of the substituents or with $E_{\text {Lumo }}$ or $E_{\text {HOMO }}$ (Table 4) for the stilbene and biphenyl derivatives. This is somewhat in contrast 
TABLE 2

\section{MUTAGENICITY OF 4-AMINOBIPHENYLS IN}

Salmonella typhimurium ${ }^{\text {a }}$

\begin{tabular}{|c|c|c|c|c|}
\hline \multirow{2}{*}{$\begin{array}{l}\text { Compounds } \\
\text { ( } \mu \text { moles) }\end{array}$} & \multicolumn{2}{|l|}{ TA98 } & \multicolumn{2}{|l|}{ TA100 } \\
\hline & $-\mathrm{S} 9$ & $+\mathrm{S} 9$ & $-\mathrm{S} 9$ & $+\$ 9$ \\
\hline \multicolumn{5}{|c|}{ 4,4'-Diaminobiphenyl } \\
\hline 0 & $29 \pm 6$ & $40 \pm$ & $121 \pm 22$ & $151 \pm 15$ \\
\hline 0.1 & $32 \pm 12$ & $56 \pm 9$ & $132 \pm 21$ & $146 \pm 23$ \\
\hline 0.25 & $24 \pm 2$ & $87 \pm 11$ & $140 \pm 11$ & $183 \pm 11$ \\
\hline 0.5 & $29 \pm 10$ & $118 \pm 10^{b}$ & $137 \pm 20$ & $181 \pm 30$ \\
\hline 1 & $24 \pm 6$ & $149 \pm 32$ & $128 \pm 15$ & $217 \pm 31^{b}$ \\
\hline 2.5 & $29 \pm 8$ & $229 \pm 19$ & $153 \pm 23$ & $258 \pm 10$ \\
\hline 5 & $27 \pm 4$ & $357 \pm 35$ & $126 \pm 11$ & $242 \pm 22$ \\
\hline 10 & $27 \pm 5$ & $472 \pm 14$ & $116 \pm 15$ & $239 \pm 25$ \\
\hline \multicolumn{5}{|c|}{ 4-Amino-4'-hydroxybiphenyl } \\
\hline 0 & $37 \pm 9$ & $47 \pm 10$ & $140 \pm 11$ & $151 \pm 17$ \\
\hline 0.01 & $37 \pm 8$ & $69 \pm 14$ & $131 \pm 11$ & $172 \pm 24$ \\
\hline 0.05 & $46 \pm 11$ & $73 \pm 11$ & $143 \pm 10$ & $191 \pm 20$ \\
\hline 0.1 & $42 \pm 14$ & $69 \pm 7$ & $139 \pm 22$ & $199 \pm 14$ \\
\hline 0.3 & $55 \pm 8$ & $100 \pm 8$ & $143 \pm 7$ & $193 \pm 9$ \\
\hline 0.5 & $47 \pm 10$ & $104 \pm 12$ & $137 \pm 2$ & $205 \pm 8$ \\
\hline 1 & $47 \pm 1$ & $165 \pm 9^{b}$ & $151 \pm 5$ & $214 \pm 8^{b}$ \\
\hline \multicolumn{5}{|c|}{ 4-Amino-4'-methylbiphenyl } \\
\hline 0 & $34 \pm 3$ & $61 \pm 14$ & $123 \pm 12$ & $156 \pm 14$ \\
\hline 0.005 & & $127 \pm 17$ & & \\
\hline 0.01 & & $177 \pm 25$ & $135 \pm 23$ & $238 \pm 26$ \\
\hline 0.033 & & $440 \pm 26$ & $129 \pm 13$ & $461 \pm 82$ \\
\hline 0.05 & $39 \pm 5$ & $569 \pm 17$ & $140 \pm 12$ & $527 \pm 6$ \\
\hline 0.066 & & $913 \pm 59^{b}$ & $128 \pm 17$ & $693 \pm 117$ \\
\hline 0.1 & $41 \pm 6$ & $1136 \pm 110$ & $137 \pm 18$ & $1061 \pm 59^{b}$ \\
\hline 0.3 & $42 \pm 9$ & $2476 \pm 74$ & & \\
\hline 0.33 & & & $113 \pm 13$ & $2375 \pm 161$ \\
\hline 0.5 & $46 \pm 7$ & $3647 \pm 322$ & & \\
\hline 1 & $49 \pm 3$ & $4075 \pm 122$ & & \\
\hline \multicolumn{5}{|c|}{ 4-Aminobiphenyl } \\
\hline 0 & $34 \pm 4$ & $65 \pm 13$ & $136 \pm 10$ & $156 \pm 10$ \\
\hline 0.01 & $40 \pm 10$ & $138 \pm 9$ & $142 \pm 9$ & $277 \pm 17$ \\
\hline 0.033 & $50 \pm 16$ & $236 \pm 22$ & $152 \pm 6$ & $614 \pm 9$ \\
\hline 0.05 & $38 \pm 5$ & $339 \pm 18$ & $155 \pm 5$ & $911 \pm 65$ \\
\hline 0.066 & $48 \pm 3$ & $280 \pm 76$ & $150 \pm 16$ & $1068 \pm 105$ \\
\hline 0.1 & $43 \pm 9$ & $661 \pm 81^{b}$ & $147 \pm 15$ & $1505 \pm 121^{b}$ \\
\hline 0.33 & $53 \pm 8$ & $1188 \pm 42$ & $145 \pm 6$ & $2596 \pm 132$ \\
\hline 0.66 & $41 \pm 7$ & & & \\
\hline 1 & $35 \pm 8$ & & & \\
\hline 3 & $35 \pm 10$ & & & \\
\hline \multicolumn{5}{|c|}{ 4-Amino-4'-chlorobiphenyl } \\
\hline 0 & $32 \pm 9$ & $45 \pm 16$ & $163 \pm 10$ & $168 \pm 28$ \\
\hline 0.005 & $33 \pm 10$ & $70 \pm 9$ & $159 \pm 19$ & $219 \pm 41$ \\
\hline 0.01 & $41 \pm 14$ & $75 \pm 9$ & $158 \pm 20$ & $211 \pm 31$ \\
\hline 0.05 & $44 \pm 16$ & $336 \pm 18$ & $159 \pm 11$ & $330 \pm 45$ \\
\hline 0.1 & $40 \pm 9$ & $645 \pm 59^{b}$ & $156 \pm 10$ & $493 \pm 68$ \\
\hline 0.3 & $46 \pm 8$ & $1511 \pm 142$ & $151 \pm 7$ & $1050 \pm 201^{b}$ \\
\hline 0.33 & $45 \pm 3$ & & & \\
\hline 0.66 & $52 \pm 22$ & & & \\
\hline
\end{tabular}

TABLE 2 (continued)

\begin{tabular}{|c|c|c|c|c|}
\hline \multirow{2}{*}{$\begin{array}{l}\text { Compounds } \\
\text { ( } \mu \text { moles })\end{array}$} & \multicolumn{2}{|l|}{ TA98 } & \multicolumn{2}{|l|}{ TA100 } \\
\hline & $-\$ 9$ & +59 & $-S 9$ & $+\mathrm{S} 9$ \\
\hline 1 & $50 \pm 4$ & & & \\
\hline 3 & $41 \pm 26$ & & & \\
\hline \multicolumn{5}{|c|}{ 4-Amino-4'-iodobiphenyl } \\
\hline 0 & $31 \pm 10$ & $50 \pm 9$ & $127 \pm 19$ & $155 \pm 16$ \\
\hline 0.005 & $32 \pm 7$ & $72 \pm 16$ & $124 \pm 20$ & $171 \pm 34$ \\
\hline 0.01 & $29 \pm 6$ & $96 \pm 19$ & $129 \pm 19$ & $179 \pm 34$ \\
\hline 0.025 & $30 \pm 13$ & $130 \pm 13$ & $124 \pm 15$ & $210 \pm 10$ \\
\hline 0.05 & $32 \pm 8$ & $472 \pm 92$ & $142 \pm 23$ & $272 \pm 69$ \\
\hline 0.1 & $34 \pm 5$ & $956 \pm 167$ & $143 \pm 14$ & $421 \pm 132$ \\
\hline 0.3 & $34 \pm 5$ & $2017 \pm 185^{b}$ & $155 \pm 8$ & $1093 \pm 106^{b}$ \\
\hline 0.5 & $38 \pm 7$ & $1663 \pm 77$ & $132 \pm 18$ & $474 \pm 25$ \\
\hline \multicolumn{5}{|c|}{ 4-Amino-4'-cyanobiphenyl } \\
\hline 0 & $38 \pm 7$ & $57 \pm 20$ & $112 \pm 11$ & $128 \pm 10$ \\
\hline 0.005 & $30 \pm 6$ & $55 \pm 6$ & & \\
\hline 0.01 & $36 \pm 8$ & $84 \pm 22$ & $112 \pm 11$ & $146 \pm 10$ \\
\hline 0.033 & $28 \pm 8$ & $120 \pm 14$ & & \\
\hline 0.05 & $46 \pm 3$ & $221 \pm 53$ & $121 \pm 9$ & $193 \pm 17^{b}$ \\
\hline 0.066 & $37 \pm 7$ & $190 \pm 17$ & & \\
\hline 0.1 & $43 \pm 12$ & $353 \pm 71$ & $113 \pm 19$ & $232 \pm 21$ \\
\hline 0.3 & $50 \pm 8$ & $909 \pm 34$ & & \\
\hline 0.5 & $59 \pm 10$ & $1358 \pm 121^{b}$ & $126 \pm 17$ & $528 \pm 82$ \\
\hline 1 & $67 \pm 8$ & $1782 \pm 245$ & $120 \pm 20$ & $687 \pm 66$ \\
\hline \multicolumn{5}{|c|}{ 4-Amino-4'-nitrobiphenyl } \\
\hline 0 & $32 \pm 9$ & $64 \pm 9$ & $124 \pm 6$ & $154 \pm 177$ \\
\hline 0.001 & $38 \pm 6$ & $76 \pm 13$ & $120 \pm 7$ & $147 \pm 14$ \\
\hline 0.005 & $49 \pm 9$ & $106 \pm 14$ & $131 \pm 20$ & $173 \pm 25$ \\
\hline 0.01 & $58 \pm 8$ & $124 \pm 20$ & $124 \pm 7$ & $172 \pm 24$ \\
\hline 0.05 & $100 \pm 11$ & $543 \pm 26$ & $132 \pm 14$ & $214 \pm 41$ \\
\hline 0.1 & $129 \pm 32$ & $1136 \pm 233^{b}$ & $140 \pm 31$ & $266 \pm 20^{b}$ \\
\hline 0.33 & $219 \pm 33$ & & & \\
\hline 0.66 & $233 \pm 27$ & & & \\
\hline 1 & $258 \pm 31$ & & & \\
\hline 3 & $311 \pm 44$ & & & \\
\hline
\end{tabular}

a Combined data from at least two independent experiments, with triplicate plating per dose and at least 5 doses per experiment.

b Indicates highest dose included in linear regression analysis.

to the correlation between mutagenicity and basicity or $E_{\text {LUMO }}$ in TA100 for 3,3'-disubstituted benzidines (You et al., 1993). However, these latter correlations were greatly dependent upon the exceptionally high mutagenicity of $3,3^{\prime}$-dinitrobenzidine relative to all the benzidine analogs we have tested to date.

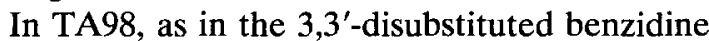
series (You et al., 1993), a correlation could be established between mutagenicity and electron- 
TABLE 3

QSAR PARAMETERS FOR THE 4-AMINOSTILBENE AND THE 4-AMINOBIPHENYL SERIES

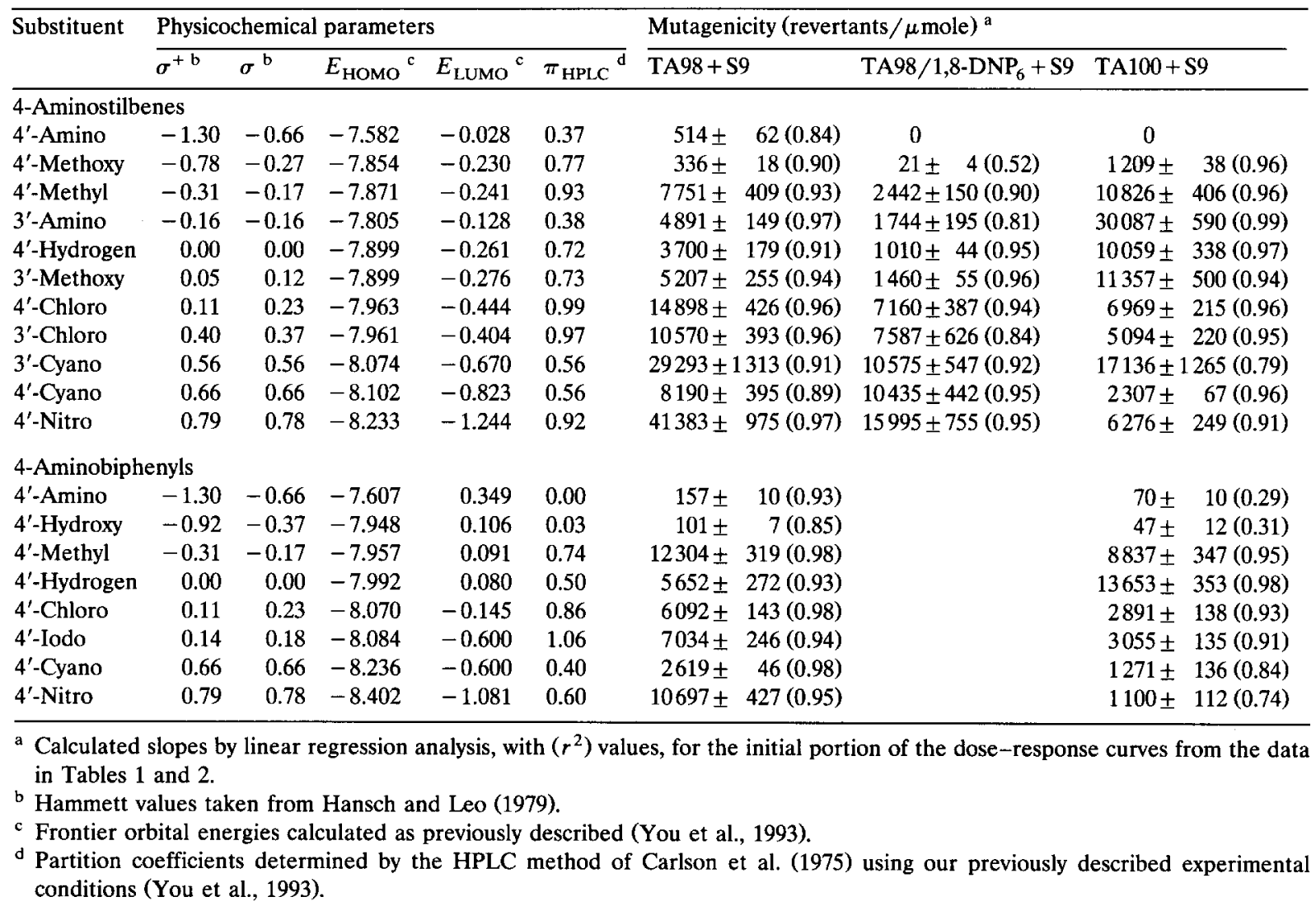

donating/withdrawing ability of a substituent for the 4-aminobiphenyls and 4-aminostilbenes (Table 4). This is also true for the stilbenes in strain TA98/1,8-DNP 6 . The correlation of electronwithdrawing capability as measured by Hammett $\sigma^{+}$values to mutagenicity is most apparent for the aminostilbenes (Fig. 1). Interestingly, when the original Hammett $\sigma$ value was used instead of $\sigma^{+}, r^{2}$ correlation values were reduced. The parameter $\sigma^{+}$reflects through-resonance inter-

\section{TABLE 4}

CORRELATION COEFFICIENTS $\left(r^{2}\right)$ FOR THE REGRESSION EQUATIONS BETWEEN MUTAGENICITY AND PHYSICOCHEMICAL PARAMETERS

\begin{tabular}{lllllll}
\hline & $\sigma^{+}$ & $\sigma$ & $E_{\text {HOMO }}$ & $E_{\text {LUMO }}$ & $\pi_{\text {HPLC }}$ & $\sigma^{+}+\pi_{\text {HPLC }}$ \\
\hline 4-Aminostilbenes & & & & & & \\
TA98 & 0.79 & 0.70 & 0.63 & 0.51 & 0.13 & 0.79 \\
TA98 1,8-DNP 6 & 0.88 & 0.74 & 0.69 & 0.42 & 0.21 & 0.90 \\
TA100 & 0.46 & 0.30 & 0.36 & 0.09 & 0.16 & 0.50 \\
4-Aminobiphenyls & & & & & & \\
TA98 & 0.61 & 0.44 & 0.39 & 0.29 & 0.75 & 0.85 \\
TA100 & 0.36 & 0.19 & 0.15 & 0.05 & 0.61 & 0.63 \\
\hline
\end{tabular}


action between an electron-rich substituent and a cationic-like reactive center that causes an extra stabilization of the ionic species (Lowry and Richardson, 1981). Compared to the correlations with $\sigma$, those with $\sigma^{+}$predict an even lower mutagenicity for the 4'-amino-, 4'-hydroxy- and 4'-methoxy-substituted compounds. Therefore, our results suggest that these strong electrondonating groups which would significantly stabilize the ultimate mutagenic species (nitrenium ions) but decrease reactivity towards DNA not only do not enhance the mutagenic potencies of these 4-aminobiphenyls and 4-aminostilbenes, but actually cause an extra reduction in mutagenicity. This effect is consistent with our analysis of mutagenicity-nitrenium ion stability and reactivity relationships for 3,3'-disubstituted benzidine derivatives (You et al., 1993).

Similar to the benzidines, mutagenicity of our stilbene derivatives in TA98 and TA98/1,8-DNP 6 does not appear to depend on hydrophobicity (Table 4), and a combination of $\sigma^{+}$and $\pi_{\text {HPLC }}$ makes no improvement over $\sigma^{+}$alone in correlations with mutagenicity. However, mutagenicity of the biphenyls in TA98 was correlated with hydrophobicity. Correlation with $\sigma^{+}$and $\pi_{\text {HPLC }}$ combined gave improved results over that with $\sigma^{+}$or $\pi_{\text {HPLC }}$ alone. Thus, a hydrophobic effect helps to explain the limitation in the mutagenicity correlation with substituent-electronic effects $\left(\sigma^{+}\right)$for the biphenyls. Hydrophobicity also plays a role for the biphenyls in strain TA100 where a weak correlation was observed. This role, however, did not extend to the stilbenes in strain TA100. Therefore, while the QSAR results of the relative mutagenicity of our benzidines and stilbenes did not depend on hydrophobicity, which is in contrast to the results of Debnath et al. (1992), our biphenyls exhibited agreement with their findings. It should be noted in making these comparisons to their results that we are concerned with series of closely related compounds as opposed to the larger series of heterogeneous amines of Debnath et al. Given below are correlation equations for the stilbenes (eqns. 1 and 2) and biphenyls (eqn. 3 ).

$$
\begin{aligned}
& \log (\mathrm{TA} 98)_{\text {stilbenes }}=0.91 \sigma^{+}+3.75 \\
& n=11, r^{2}=0.79 \\
& \log \left(\mathrm{TA} 98 / 1,8-\mathrm{DNP}_{6}\right)_{\text {stilbenes }}=1.61 \sigma^{+}+3.19
\end{aligned}
$$
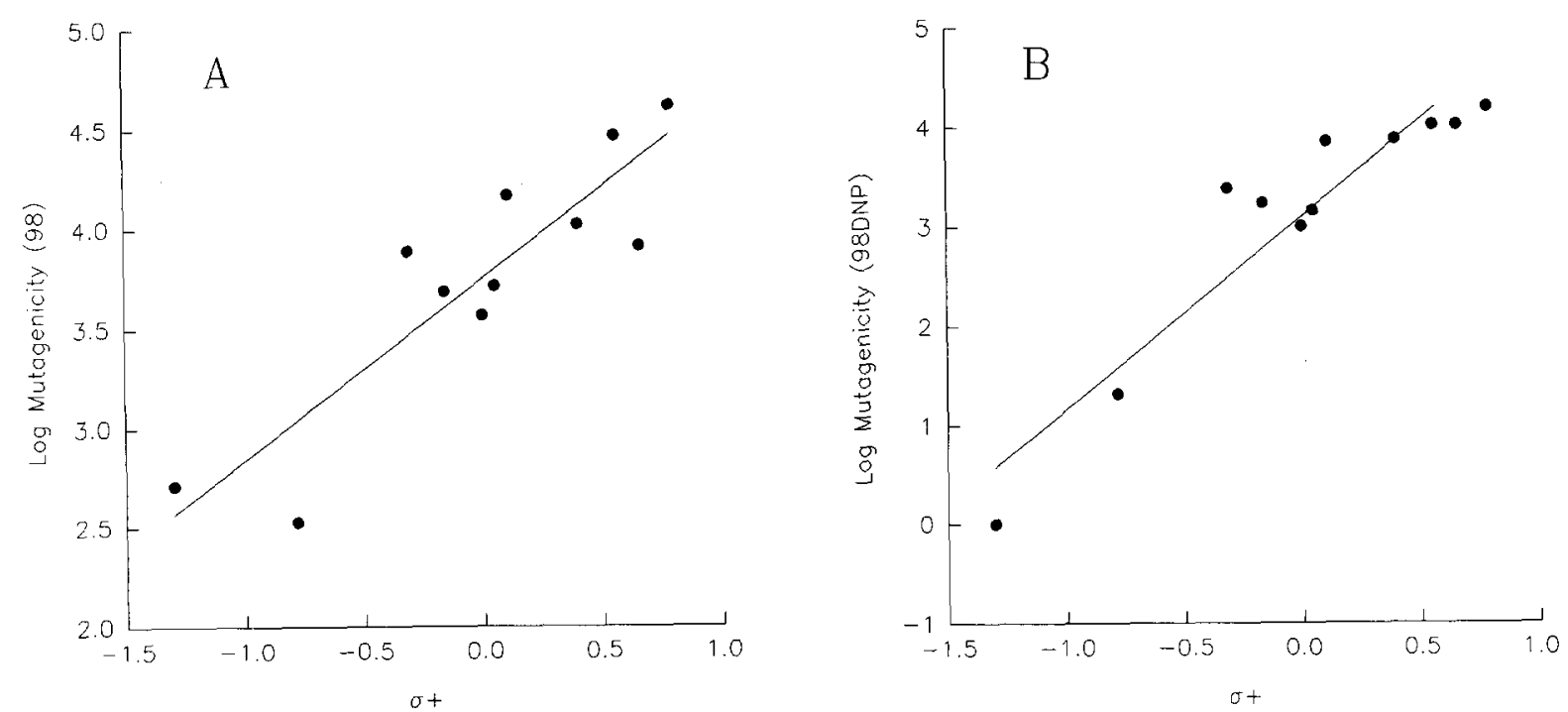

Fig. 1. Relationship between electron-withdrawing capability, as measured by Hammett $\sigma^{+}$values and mutagenicity towards Salmonella tester strains TA98 (A) and TA98/1,8-DNP $(\mathrm{B})$. 
$n=11, r^{2}=0.88$

$\log (\text { TA98 })_{\text {biphenyls }}=0.46 \sigma^{+}+1.36 \pi_{\text {HPLC }}+2.72$

$n=8, r^{2}=0.85$

These correlations with $\sigma^{+}$were more pronounced than the correlations of mutagenicity with $E_{\text {LUMO }}$ or $E_{\text {HOMO }}$ for the stilbenes and there was little correlation to frontier orbital energies for the biphenyls (Table 4). Also, similar to the combination of $\pi_{\text {HPLC }}$ with $\sigma^{+}$, correlation of mutagenicity with $E_{\text {HOMO }}$ (or $E_{\text {LUMO }}$ ) and $\pi_{\text {HPLC }}$ did not significantly improve the correlation coefficients for the stilbenes.

In comparing the differences in QSAR results between strains TA98 and TA100, it should be noted that there is a correlation between strains for the biphenyl series $\left(r^{2}=0.83\right)$ but not for the aminostilbenes $\left(r^{2}=0.41\right)$. Since the only significant difference between the two strains is in the target DNA sequence, where TA98 detects frameshift mutations and TA100 is sensitive to base-pair substitutions, Debnath et al. (1992) postulate that the differences in mutagenic response relate to how the activated amine reacts with DNA. Debnath et al. found that aromatic systems with three of more fused rings demonstrated enhanced frameshift mutagenicity and suggest that this effect may be attributable to intercalation of larger compounds into DNA. We have previously shown for benzidine analogs (Messerly et al., 1987), including two compounds with fused rings (Sinsheimer et al., 1992), that, in general, planar compounds, where intercalation might be facilitated, are more mutagenic in TA98 than in TA100. While this greater mutagenicity in TA98 is also found for the benzidines (You et al., 1993) and in the present study for all of the biphenyls, except for 4-aminobiphenyl, it is not true for the aminostilbenes. That is, for the parent 4-aminostilbene and 4 of 5 derivatives with electron-donating substituents, but not for compounds with electron-withdrawing groups, there is greater mutagenicity in TA100 than in TA98. It is of interest to question whether or not these differences in substituent effects contribute to differences in mutational sites, and ultimately strain specificity, such as those described by Rosenkranz et al. (1985).

Mechanisms of hepatic DNA binding for 4aminobiphenyl and many other aromatic amines have been studied (Beland and Kadlubar, 1990). A generally accepted pathway for these aromatic amines is enzymatic $N$-hydroxylation, acid catalyzed $\mathrm{N}-\mathrm{O}$ bond cleavage of the hydroxylamine, or ester formation (in particular, $O$-acetylation and $O$-sulfonation) before this $\mathrm{N}-\mathrm{O}$ cleavage and finally DNA binding with the resulting nitrenium ion (Beland and Kadlubar, 1990; Helmick and Novak, 1991). Our structure-activity relationship similarities with TA98 between the biphenyls and the stilbenes and between these two series and the benzidine series (You et al., 1993) may well be caused by their common bacterial DNA-binding features. As discussed in the benzidine paper, enhanced stability of the intermediate $N$-hydroxylamine with increased electron-withdrawing substituents but with greater reactivity of the ultimate mutagenic nitrenium ion serve as the rational for our observed results. In addition, as evidenced by the correlations with hydrophobicity of the biphenyls and as noted by Debnath et al. (1992), partition coefficients can become a factor in correlating structure to mutagenicity.

\section{In vivo results}

All of the compounds in the biphenyl and stilbene series showed a significant increase in aberrations at $100 \mathrm{mg} / \mathrm{kg}$ compared to the negative control (Table 5). A limitation of our CA testing is that, at the relatively high dose required to establish differences over the negative control and among compounds, there was evidence of both acute toxicity and low mitotic indices for several of these amines. The mean percentage of aberrant cells could not be correlated to substituent-electronic effects or the frontier orbital energies of the compounds as for the in vitro assay. Nor could it be explained by hydrophobic effects. As was true in the 3,3'-disubstituted benzidine series (You et al., 1993), the low mutagenicity of benzidine in vitro was not predictive of its greater genotoxicity in vivo. However, a parallel situation did not exist in comparing the in vitro and in vivo genotoxicity of $4,4^{\prime}$-diaminostilbene, which exhibited low genotoxicity in both 
TABLE 5

CHROMOSOMAL ABERRATIONS INDUCED BY 4-AMINOSTILBENES AND 4-AMINOBIPHENYLS IN BONE-MARROW CELLS OF MICE

\begin{tabular}{|c|c|c|c|c|c|}
\hline \multirow{2}{*}{$\begin{array}{l}\text { Treatment } \\
(\mathrm{mg} / \mathrm{kg})\end{array}$} & \multirow[t]{2}{*}{ Gaps $^{a}$} & \multicolumn{2}{|c|}{ Aberrations/cell } & \multirow{2}{*}{$\begin{array}{l}\text { Aberrant cells }(\%)^{b} \\
(\text { mean } \pm \text { S.D. })^{c}\end{array}$} & \multirow{2}{*}{$\begin{array}{l}\text { Mitotic indices (\%) } \\
(\text { mean } \pm \text { S.D.) }\end{array}$} \\
\hline & & $\begin{array}{l}\text { Chromatid } \\
\text { type }\end{array}$ & $\begin{array}{l}\text { Chromosome } \\
\text { type }\end{array}$ & & \\
\hline \multicolumn{6}{|l|}{ Solvent control } \\
\hline DMSO & 3.00 & 0.010 & 0.000 & $1.00 \pm 0.82$ & $3.51 \pm 0.30$ \\
\hline \multicolumn{6}{|l|}{ Positive control } \\
\hline DMBA (100 mg/kg) & 11.25 & 0.105 & 0.023 & $13.25 \pm 0.96 * *$ & $2.13 \pm 0.12 * *$ \\
\hline \multicolumn{6}{|l|}{ 4-Aminostilbenes } \\
\hline 25 & 5.25 & 0.023 & 0.000 & $2.25 \pm 0.50 *$ & $2.85 \pm 0.38 *$ \\
\hline 100 & 4.50 & 0.025 & 0.000 & $2.50 \pm 1.00 *$ & $2.58 \pm 0.30 * *$ \\
\hline \multicolumn{6}{|l|}{ 4'-Methoxy } \\
\hline 25 & 2.50 & 0.023 & 0.000 & $2.25 \pm 0.50 *$ & $3.20 \pm 0.44$ \\
\hline 100 & 4.50 & 0.038 & 0.000 & $3.75 \pm 0.96 * *$ & $2.82 \pm 0.30 * *$ \\
\hline \multicolumn{6}{|l|}{$4^{\prime}$-Methyl } \\
\hline 25 & 2.00 & 0.030 & 0.000 & $3.00 \pm 0.82 * *$ & $2.33 \pm 0.33 * *$ \\
\hline $100^{d}$ & 6.33 & 0.080 & 0.017 & $8.67 \pm 1.53 * *$ & $1.93 \pm 0.42 * *$ \\
\hline \multicolumn{6}{|l|}{ 3'-Amino } \\
\hline 25 & 2.50 & 0.043 & 0.000 & $3.75 \pm 0.96 * *$ & $2.52 \pm 0.32 * *$ \\
\hline 100 & 5.50 & 0.050 & 0.000 & $5.00 \pm 0.82 * *$ & $2.10 \pm 0.22 * *$ \\
\hline \multicolumn{6}{|l|}{ 4'-Hydrogen } \\
\hline 10 & 3.00 & 0.030 & 0.000 & $2.75 \pm 0.50 * *$ & $2.77 \pm 0.38 *$ \\
\hline 25 & 5.25 & 0.085 & 0.013 & $8.50 \pm 1.29 * *$ & $2.65 \pm 0.35 * *$ \\
\hline $100^{d}$ & 11.00 & 0.303 & 0.023 & $20.33 \pm 2.08 * *$ & $1.50 \pm 0.24 * *$ \\
\hline \multicolumn{6}{|l|}{ 3'-Methoxy } \\
\hline 25 & 3.50 & 0.038 & 0.003 & $3.75 \pm 0.50 * *$ & $2.49 \pm 0.39 * *$ \\
\hline 100 & 4.50 & 0.060 & 0.008 & $6.75 \pm 1.50 * *$ & $2.22 \pm 0.28 * *$ \\
\hline \multicolumn{6}{|l|}{$4^{\prime}$-Chloro } \\
\hline 25 & 2.50 & 0.020 & 0.000 & $2.00 \pm 0.82$ & $2.66 \pm 0.09 * *$ \\
\hline 100 & 6.25 & 0.028 & 0.005 & $3.25 \pm 0.50 * *$ & $2.28 \pm 0.08 * *$ \\
\hline \multicolumn{6}{|l|}{ 3'-Chloro } \\
\hline 25 & 4.25 & 0.035 & 0.005 & $4.00 \pm 0.82 * *$ & $2.36 \pm 0.44 * *$ \\
\hline $100^{\mathrm{e}}$ & 7.00 & 0.060 & 0.010 & $7.00 * *$ & $1.63 * *$ \\
\hline \multicolumn{6}{|l|}{$3^{\prime}$-Cyano } \\
\hline 25 & 6.00 & 0.058 & 0.000 & $5.25 \pm 0.96 * *$ & $2.46 \pm 0.38 * *$ \\
\hline 100 & 8.50 & 0.145 & 0.000 & $12.75 \pm 0.96 * *$ & $2.17 \pm 0.15 * *$ \\
\hline \multicolumn{6}{|l|}{$4^{\prime}$-Cyano } \\
\hline 25 & 2.25 & 0.018 & 0.000 & $1.75 \pm 0.50$ & $2.47 \pm 0.36 * *$ \\
\hline 100 & 3.50 & 0.028 & 0.000 & $2.75 \pm 0.96 *$ & $2.31 \pm 0.31 * *$ \\
\hline \multicolumn{6}{|l|}{$4^{\prime}$-Nitro } \\
\hline 25 & 4.50 & 0.028 & 0.000 & $2.75 \pm 0.50 * *$ & $2.31 \pm 0.31 * *$ \\
\hline 100 & 3.75 & 0.043 & 0.008 & $5.00 \pm 0.82 * *$ & $1.95 \pm 0.29 * *$ \\
\hline \multicolumn{6}{|l|}{$\begin{array}{l}\text { 4-Aminobiphenyls } \\
\text { 4'-Amino }\end{array}$} \\
\hline 100 & 6.25 & 0.043 & 0.080 & $5.00 \pm 0.82 * *$ & $2.63 \pm 0.33 * *$ \\
\hline
\end{tabular}


TABLE 5 (continued)

\begin{tabular}{|c|c|c|c|c|c|}
\hline \multirow{2}{*}{$\begin{array}{l}\text { Treatment } \\
(\mathrm{mg} / \mathrm{kg})\end{array}$} & \multirow[t]{2}{*}{ Gaps $^{a}$} & \multicolumn{2}{|c|}{ Aberrations/cell } & \multirow{2}{*}{$\begin{array}{l}\text { Aberrant cells (\%) } \\
\text { (mean } \pm \text { S.D. }^{\mathrm{c}}\end{array}$} & \multirow{2}{*}{ 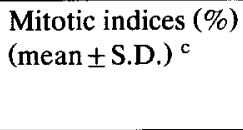 } \\
\hline & & $\begin{array}{l}\text { Chromatid } \\
\text { type }\end{array}$ & $\begin{array}{l}\text { Chromosome } \\
\text { type }\end{array}$ & & \\
\hline $4^{\prime}$-Hydroxy & & & & & \\
\hline 100 & 3.75 & 0.025 & 0.003 & $2.75 \pm 0.50 * *$ & $2.82 \pm 0.25 * *$ \\
\hline $\begin{array}{l}4^{\prime} \text {-Methyl } \\
100\end{array}$ & 3.75 & 0.038 & 0.003 & $4.00 \pm 0.82 * *$ & $2.59 \pm 0.22 * *$ \\
\hline 4'-Hydrogen & & & & & \\
\hline $100^{\mathrm{d}}$ & $\begin{array}{l}3.25 \\
6.67\end{array}$ & $\begin{array}{l}0.028 \\
0.067\end{array}$ & $\begin{array}{l}0.000 \\
0.003\end{array}$ & $\begin{array}{l}2.75 \pm 0.96 * \\
7.00 \pm 1.00 * *\end{array}$ & $\begin{array}{l}3.62 \pm 0.37 \\
1.69 \pm 0.25 * *\end{array}$ \\
\hline $\begin{array}{l}4^{\prime} \text {-Chloro } \\
25 \\
100^{\mathrm{f}}\end{array}$ & 3.00 & 0.033 & 0.000 & $3.00 \pm 0.82 * *$ & $2.31 \pm 0.16 * *$ \\
\hline $\begin{array}{l}4^{\prime} \text {-Iodo } \\
100^{\mathrm{g}}\end{array}$ & 2.50 & 0.030 & 0.000 & $3.00 \pm 0.00 * *$ & $2.05 \pm 0.11 * *$ \\
\hline $\begin{array}{l}4^{\prime} \text {-Cyano } \\
100\end{array}$ & 3.50 & 0.028 & 0.000 & $2.75 \pm 0.50 * *$ & $2.77 \pm 0.49 *$ \\
\hline $\begin{array}{l}4^{\prime}-\text { Nitro } \\
100\end{array}$ & 4.00 & 0.033 & 0.008 & $4.00 \pm 0.82 * *$ & $1.77 \pm 0.24 * *$ \\
\hline
\end{tabular}

a Total chromatid and chromosome gaps at each concentration per 100 cells were recorded but not included as aberrations.

b Cells with at least 1 aberration. Results are for 4 animals at each concentration ( 100 cells /animal) except where noted.

c Results at each concentration were compared to those of the control using Dunnett's test ( ${ }^{*} P<0.05$ and ${ }^{* *} P<0.01$ ).

d 1 of the 4 animals died at this concentration.

e 3 of the 4 animals died at this concentration.

f All 4 animals died at this concentration.

$\mathrm{g} 2$ of the 4 animals died at this concentration.

the in vitro and in vivo tests. The moderate in vivo genotoxicity for the nitro compounds in both the benzidine and stilbene series was not reflective of their high in vitro mutagenicity.

All of the 3 'substituted 4-aminostilbenes showed substantially higher in vivo genotoxicity than their 4'-substituted counterparts. This was especially pronounced for the comparison of the cyano derivatives. The high in vivo genotoxicity of the two parent compounds, 4-aminobiphenyl and 4-aminostilbene, would not have been predicted from their in vitro mutagenicity results. The CA values for 4-aminostilbene remain the highest for any of the benzidine analogs we have tested to date and indeed are at least equal for that of our positive control, the established carcinogen, 7,12-dimethylbenz $[a]$ anthracene.

\section{Acknowledgement}

This investigation was supported by grant ROPES 05047 from the National Institute of Environmental Health Sciences, DHHS.

\section{References}

Ashley, J.N., H.J. Barber, A.J. Ewins, G. Newbery and A.D.H. Self (1942) Chemotherapeutic comparison of the trypanocidal action of some aromatic diamidines, J. Chem. Soc., 103-116.

Beland, F.A., and F.F. Kadlubar (1985) Formation and persistence of arylamine DNA adducts in vivo, Environ. Health Perspect., 62, 19-30.

Beland, F.A., and F.F. Kadlubar (1990) Metabolic activation and DNA adducts of aromatic amines and nitroaromatic hydrocarbons, in: C.S. Cooper and P.L. Grover (Eds.), 
Chemical Carcinogenesis and Mutagenesis I, Springer, Berlin, pp. 267-325.

Byron, D.J., G.W. Gray and R.C. Wilson (1966) The effects of $3^{\prime}$ and $4^{\prime}$ substituents on the ionization constants of biphenyl-4-carboxylic acid and 4-aminobiphenyl, J. Chem. Soc. (C), 831-836.

Carlson, R.M., R.E. Carison and H.L. Kopperman (1975) Determination of partition coefficients by liquid chromatography, J. Chromatog., 107, 219-223.

Czerwinska-Fejgin, E., and W. Polaczkowa (1967) Conjugation in the $o$-terphenyl systems, II. Dissociation constants of 4-amino-4'-substituted biphenyl and 4-amino-4'-substituted-o-terphenyl derivatives, Rocz. Chem., 41, 17591765.

Debnath, A.K., G. Debnath, A.J. Shusterman and C. Hansch (1992) A QSAR investigation of the role of hydrophobicity in regulating mutagenicity in the Ames test, 1 . Mutagenicity of aromatic and heteroaromatic amines in Salmonella typhimurium TA98 and TA100, Environ. Mol. Mutagen., $19,37-52$.

Giri, A.K., E.A. Messerly and J.E. Sinsheimer (1989) Sisterchromatid exchange and chromosome aberrations for 4 aliphatic epoxides in mice, Mutation Res., 224, 253-261.

Grieve, W.S.M., and D.H. Hey (1932) Subtitution in compounds containing two or more phenyl groups, Part I. The nitration of 4-methyldiphenyl, J. Chem. Soc., 1888-1894

Hanna, P.E., R.E. Gammans, R.D. Sehon and M.-K. Lee (1980) Metabolic $N$-hydroxylation, Use of substituent variation to modulate the in vitro bioactivation of 4-acetamidostilbenes, J. Med. Chem., 23, 1038-1044.

Hansch, C., and A. Leo (1979) Substituent Constants for Correlation Analysis in Chemistry and Biology, Wiley, New York.

Harley-Mason, J., and F.G. Mann (1940) Quaterphenyl, Part I. Some dihydroxy-derivatives, J. Chem. Soc., 1379-1385.

Helmick, J.S., and M. Novak (1991) Nucleophilic substitution at nitrogen and carboxyl carbon of $N$-aryl- $O$-pivaloylhydroxylamines in aqueous solution: competition with $S_{N} 1$ solvolysis of model carcinogens, J. Org. Chem., 56, 29252927.

Kennelly, J.C., C.A. Stanton and C.N. Martin (1984) The effect of acetyl-CoA supplementation on the mutagenicity of benzidines in the Ames assay, Mutation Res., 137, 39-45.

Lowry, H.L., and K.S. Richardson (1981) Mechanism and theory in organic chemistry, Harper and Row, New York, pp. $130-136$.

Maron, D.M., and B.N. Ames (1983) Revised methods for the Salmonella mutagenicity test, Mutation Res., 113, 173-215.
McCoy, E.C., H.S. Rosenkranz and R. Mermelstein (1981) Evidence for the existence of a family of bacterial nitroreductases capable of activating nitrated polycylics to mutagens, Environ. Mutagen., 3, 421-427.

Messerley, E.A., J.E. Fekete, D.R. Wade and J.E. Sinsheimer (1987) Structure-mutagenicity relationships of benzidine analogues, Environ. Mol. Mutagen., 10, 263-274.

Natarajan, A.T., and G. Obe (1986) How do in vivo mammalian assays compare to in vitro assays in their ability to detect mutations? Mutation Res., 167, 189-201.

Nohara, M., T. Hirayama, Y. Fujioka, S. Ozasa, E. Ibuki and S. Fukui (1985) Relationship between mutagenic potency in Salmonella typhimurium and chemical structure of amino- and nitro-substituted biphenyls, Mutation Res., 149, 9-15.

Preston, R.J., B.J. Dean, S. Galloway, H. Holden, A.F. McFee and M. Shelby (1987) Mammalian in vivo cytogenetic assays. Analysis of chromosome aberrations in bone marrow cells, Mutation Res., 189, 157-165.

Rosenkranz, H.S., and R. Mermelstein (1983) Mutagenicity and genotoxicity of nitroarenes, All nitro-containing chemicals were not created equal, Mutation Res., 114, 217-267.

Rosenkranz, H.S., E.C. McCoy, M. Frierson and G. Klopman (1985) The role of DNA sequence and structure of the electrophile on the mutagenicity of nitroarenes and arylamine derivatives, Environ. Mutagen., 7, 645-653.

Saito, K., A. Shinohara, T. Kamataki and R. Kato (1985) Metabolic activation of mutagenic $N$-hydroxyarylamines by $O$-acetyltransferase in Salmonella typhimurium TA98, Arch. Biochem. Biophys., 239, 286-295.

Shelby, M.D. (1988) The genetic toxicity of human carcinogens and its implications, Mutation Res., 204, 3-15.

Sinsheimer, J.E., B.H. Hooberman, S.K. Das, M.D. Brezzell and $Z$. You (1992) The in vivo and in vitro genotoxicity of aromatic amines in relationship to the genotoxicity of benzidine, Mutation Res., 268, 255-262.

Veschamber, H., and A. Kergomard (1966) La transmission des effets électroniques dans les trans-stilbènes substitués, Bull. Soc. Chim. (France), 336-342.

You, Z., M.D. Brezzell, S.K. Das, M.C. Espada-Torre, B.H Hooberman and J.E. Sinsheimer (1993) Ortho-substituent effects on the in vitro and in vivo genotoxicity of benzidine derivatives, Mutation Res., 319, 19-30.

Zheltov, A.Y., V.Y. Rodionov and B.I. Stepanov (1970) Investigations on aromatic disulfides, III. Basicity and structure of substituted 4- and 5-aminobiphenylene-2,2'-disulfide, J. Org. Chem. (U.S.S.R.), 6, 2573-2578. 